\title{
Unidirectional Rotary Motion in Isotopically Chiral Molecular Motors: A Computational Analysis
}

\author{
Jun Wang,* Baswanth Oruganti, and Bo Durbeej* \\ Cite This: https://dx.doi.org/10.1021/acs.orglett.0c02436 \\ Read Online
}

ACCESS |

Wlll Metrics \& More

Article Recommendations

Supporting Information

ABSTRACT: Molecular dynamics simulations are performed to explore if isotopic chirality can induce unidirectional rotary motion in molecular motors operated through double-bond photoisomerizations. Using a highquantum yield motor featuring a chemically asymmetric carbon atom as reference, it is found that isotopically chiral counterparts of this motor sustain such motion almost equally well. Overall, the study reveals a previously unexplored role for isotopic chirality in the design of rotary molecular motors.

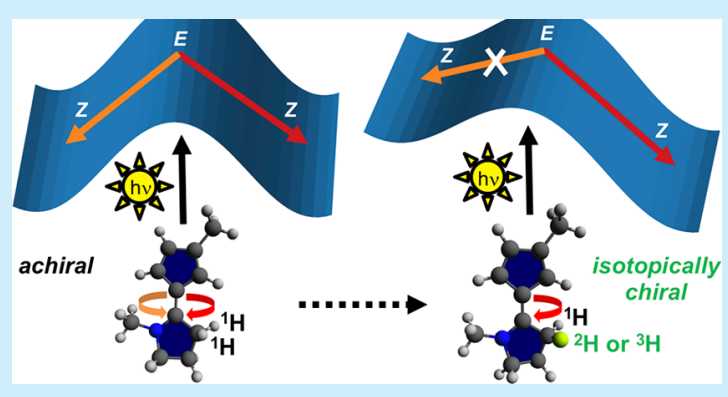

$\mathrm{D}$ ouble-bond photoisomerization is the reaction upon which many different types of synthetic molecular motors rely to produce $360^{\circ}$ unidirectional rotary motion through the absorption of ultraviolet (UV) or visible light, with examples such as overcrowded-alkene, ${ }^{1}$ hemithioindigo, ${ }^{2}$ dibenzofulvene, $^{3}$ and $\mathrm{N}$-alkyl-imine ${ }^{4}$ motors. Key to exerting control of the photoinduced rotation of such motors is making the excitedstate dynamics asymmetric with respect to clockwise (CW) and counterclockwise (CCW) directions for the photoisomerizations. This asymmetry is usually invoked by introducing chirality into the motors, for example, by the presence of a stereocenter; however, permanent point chirality is not an absolute requirement in this regard. In fact, the occurrence of a chiral-like feature in the form of a pseudoasymmetric carbon atom has been shown to be sufficient for an overcrowded-alkene motor to sustain unidirectional rotary motion. ${ }^{5}$ Similarly, the incorporation of an axially chiral trityl moiety has been found to have the same effect for dibenzofulvene motors. ${ }^{3}$

Furthermore, on the basis of quantum chemical calculations and non-adiabatic molecular dynamics (NAMD) simulations, ${ }^{6}$ it has been demonstrated that the $\mathrm{CW}$ or $\mathrm{CCW}$ rotary direction of motors featuring Schiff-base and cyclohexenylidene motifs can be controlled by the intrinsic asymmetry of the ringpuckered cyclohexenylidene. ${ }^{7}$ In addition, besides exploiting chiral-like molecular features, another approach to direct the rotary motion of molecular motors is through the chirality of the photons with which the motors are powered. ${ }^{8}$

In this work, we perform NAMD simulations to explore, for the first time, whether isotopic chirality ${ }^{9}$ can be used to achieve the double-bond photoisomerization asymmetry required for light-driven molecular motors to exhibit unidirectional rotary motion. This form of chirality results from isotopic substitution of an otherwise achiral species and has been manifested in many experimental contexts; however, its consequences are less readily detectable than those associated with a tetrahedral, asymmetric carbon atom bonded to four different groups. The first measurement of optical activity of an isotopically chiral molecule was reported in $1949^{10}$ and was subsequently followed by other measurements ${ }^{9 a, 11}$ and, in 2006, the first theoretical analysis. ${ }^{12}$ Furthermore, supramolecular chirality induced by isotopic substitution has been documented in self-assembly studies, ${ }^{13}$ and it has been found possible to trigger asymmetric autocatalysis (the process by which a chiral reaction product acts as a catalyst to produce more of itself ${ }^{14}$ ) through isotopic substitution of hydrogen $\left({ }^{2} \mathrm{H} /{ }^{1} \mathrm{H}\right),{ }^{15}$ carbon $\left({ }^{13} \mathrm{C} /{ }^{12} \mathrm{C}\right),{ }^{16}$ oxygen $\left({ }^{18} \mathrm{O} /{ }^{16} \mathrm{O}\right),{ }^{17}$ and nitrogen $\left({ }^{15} \mathrm{~N} /{ }^{14} \mathrm{~N}\right){ }^{18}$ atoms. In addition, recently the first asymmetric synthesis of ${ }^{13} \mathrm{C} /{ }^{12} \mathrm{C}$ isotopically chiral hydrocarbons was reported. ${ }^{19}$

The motor design evaluated in this work is based on a previous design ${ }^{20}$ shown in Figure 1a. This design features a cyclopentadiene motif connected by an $E / Z$-isomerizable olefinic bond to an electron-donating $N$-methylpyrrolidine motif, which is chiral due to the presence of an additional methyl group at the C5 position. Specifically, the idea underlying this design is that the modest photoisomerization quantum yields that limit the efficiency of many light-driven molecular motors, ${ }^{21}$ can be improved by a motor in which the cleavage of the isomerizing $\pi$-bond in the excited state is coupled to the transformation of the non-aromatic cyclopentadiene into an aromatic cyclopentadienyl anion. ${ }^{20}$ As confirmed by quantum chemical calculations, such a transformation does indeed take

Received: July 21, 2020 
(a)

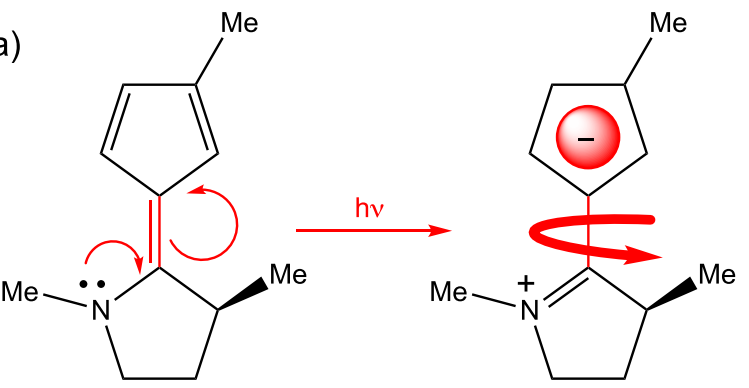

(b)

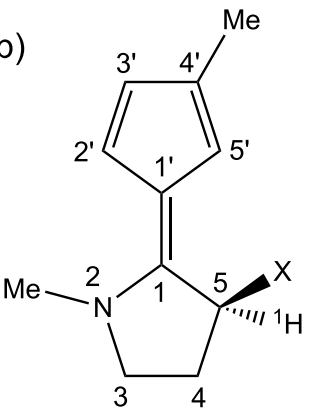

1: $\mathrm{X}={ }^{1} \mathrm{H}$ (achiral)

2: $\mathrm{X}={ }^{2} \mathrm{H}$ (isotopically chiral)

3: $\mathrm{X}={ }^{3} \mathrm{H}$ (isotopically chiral)

4: $X=M e$ (chemically chiral)

$\theta=\mathrm{N} 2-\mathrm{C} 1-\mathrm{C}^{\prime}-\mathrm{C} 5^{\prime}$

$\theta^{\prime}=\mathrm{C} 5-\mathrm{C} 1-\mathrm{C}^{\prime}-\mathrm{C} 2^{\prime}$

$\omega=1 / 2\left(\theta+\theta^{\prime}\right)$

$\alpha=\mathrm{C} 1-\mathrm{N} 2-\mathrm{C} 5-\mathrm{C} 1^{\prime}$

$\alpha^{\prime}=\mathrm{C} 1^{\prime}-\mathrm{C} 2^{\prime}-\mathrm{C} 5^{\prime}-\mathrm{C} 1$

Figure 1. (a) Heterolytic $\pi$-bond cleavage in the $S_{2}$ state of a molecular motor promoted by the onset of excited-state aromaticity. ${ }^{20}$ (b) Molecules (shown in their $E$ isomeric form with respect to the central olefinic bond) studied in this work and definitions of key dihedral angles.

place in the bright second excited singlet state $\left(\mathrm{S}_{2}\right)$ of the motor in which the $E / Z$ photoisomerizations occur, through electron donation from the $N$-methylpyrrolidine motif. ${ }^{20 a}$ Furthermore, NAMD simulations predicted that this process enables the motor to achieve high quantum yields of $\sim 75 \%{ }^{20 a}$

Deriving from the chiral motor design in Figure 1a, which is hereafter denoted 4, the systems considered in this work are shown in Figure 1b. In 1, the C5 methyl group of $\mathbf{4}$ is replaced by ${ }^{1} \mathrm{H}$, whereby this molecule becomes achiral. In 2 and 3, the methyl is replaced by ${ }^{2} \mathrm{H}$ and ${ }^{3} \mathrm{H}$, respectively, and consequently, these molecules are isotopically chiral. Overall, 1-3 are sufficiently small to allow for the type of demanding NAMD simulations that will be presented herein. Moreover, because of the large mass ratios of ${ }^{3} \mathrm{H}$ and ${ }^{2} \mathrm{H}$ over ${ }^{1} \mathrm{H}$ (compared to isotopes of other elements), these systems appear to be ideal for exploring whether isotopic chirality can be exploited to accomplish unidirectional rotary motion in molecular motors.

First, as a control that $\mathbf{1 - 3}$ retain the electronic features of $\mathbf{4}$, the complete active space second-order perturbation theory $(\text { CASPT2 })^{22}$ and the approximate coupled-cluster singles and doubles $(\mathrm{CC} 2)^{23}$ methods were used to calculate vertical transition energies from the ground state $\left(S_{0}\right)$ to the two lowest excited singlet states $\left(S_{1}\right.$ and $\left.S_{2}\right)$ of the $E$ and $Z$ isomers of $\mathbf{1 - 4}$. For the former method, and for all other CAS-based modeling in this work, an $(8,7)$ active space comprising the full $\pi$-system of the cyclopentadiene-pyrrolidine skeleton and the nitrogen lone pair of 1-4 was employed. Notably, within the standard adiabatic Born-Oppenheimer (BO) approximation used for the calculations, nuclear masses do not enter the electronic Hamiltonian, which means that (presumably small) electronic differences among 1-3 are not accounted for. The results of the calculations, carried out with the cc-pVTZ basis set (used for all calculations in this work except where otherwise noted), are listed in Table S1. Pleasingly, both CASPT2 and CC2 predict that the $S_{1}$ and $S_{2}$ states of $\mathbf{1 - 3}$ are very similar to those of $\mathbf{4}$, with
$S_{1}$ being a dark state and $S_{2}$ a bright state (oscillator strength of 0.6-0.7) populated by absorption of UV photons.

Subsequently, as a test of whether excitation to the $S_{2}$ state triggers $E / Z$ photoisomerizations of $1-3$ around the central olefinic bond, minimum energy path (MEP) calculations starting from the vertically excited $S_{2}$ Franck-Condon (FC) points of their $E$ and $Z$ isomers were performed by using the complete active space self-consistent field (CASSCF) method. ${ }^{24}$ The results are presented in Figure 2.

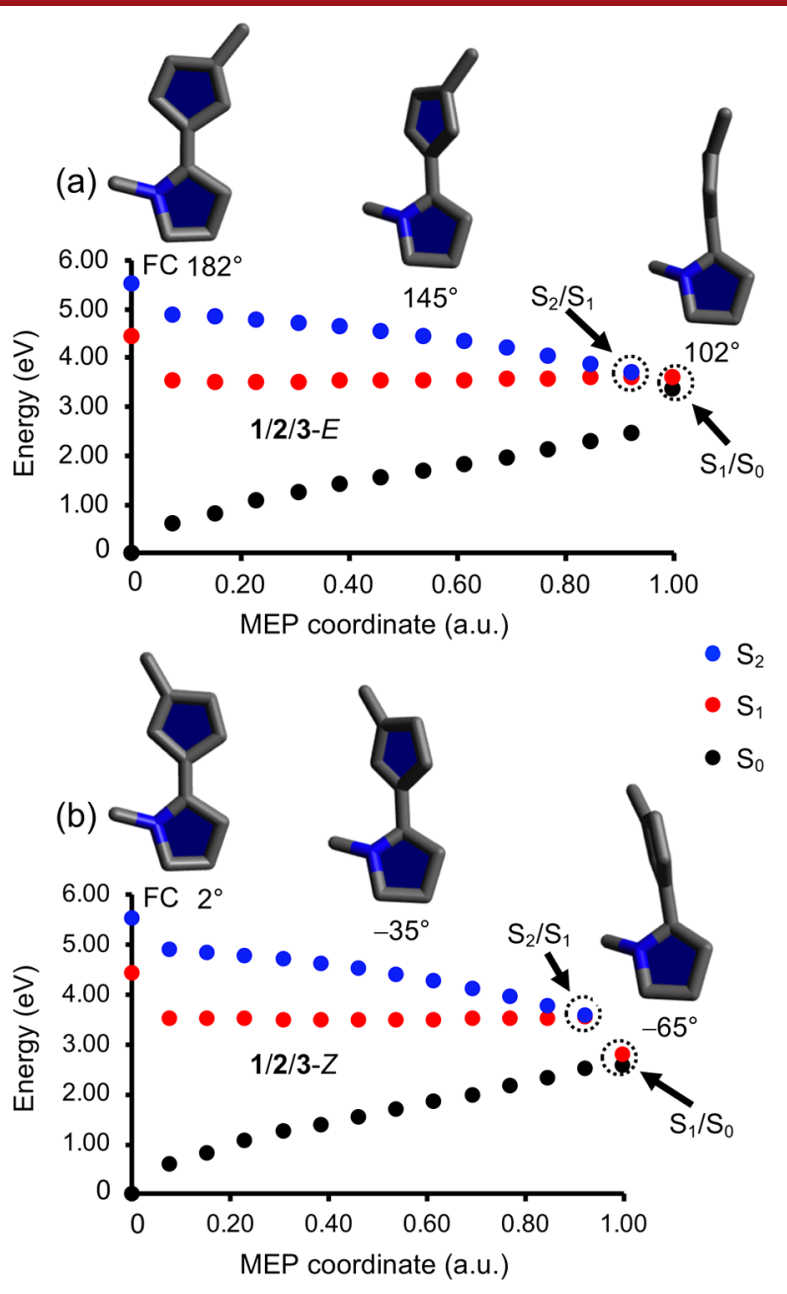

Figure 2. MEPs from the $\mathrm{S}_{2} \mathrm{FC}$ points of the (a) $E$ and (b) $Z$ isomers of 1-3. Also shown are molecular geometries at the FC point, the seventh MEP point, and the final MEP point, as well as the corresponding $\omega$ dihedral angles (defined in Figure 1b). Encircled points are presumably close to conical intersection seams.

From the MEPs in Figure 2, it is clear that the $S_{2}$ state can evolve through a torsional reaction coordinate that describes $E$ / $Z$ photoisomerizations, as shown by the pronounced and barrierless changes in the $\omega$ dihedral angle (defined in Figure 1b) along the MEPs. Furthermore, from the localization of molecular geometries for both isomers with small $S_{2} / S_{1}(\sim 1-2$ $\left.\mathrm{kcal} \mathrm{mol}^{-1}\right)$ and $S_{1} / S_{0}\left(5 \mathrm{kcal} \mathrm{mol}^{-1}\right)$ energy gaps, the MEP calculations also detect possible funnels for the photoisomerization of the $E(Z)$ species to form the ground-state $Z(E)$ species, by successively reaching presumed $S_{2} / S_{1}$ and $S_{1} / S_{0}$ conical intersection seams.

With regard to the character of the $S_{2}$ state, in turn, Figure S2 summarizes geometric ${ }^{25}$ and electronic ${ }^{26}$ aromaticity indices 

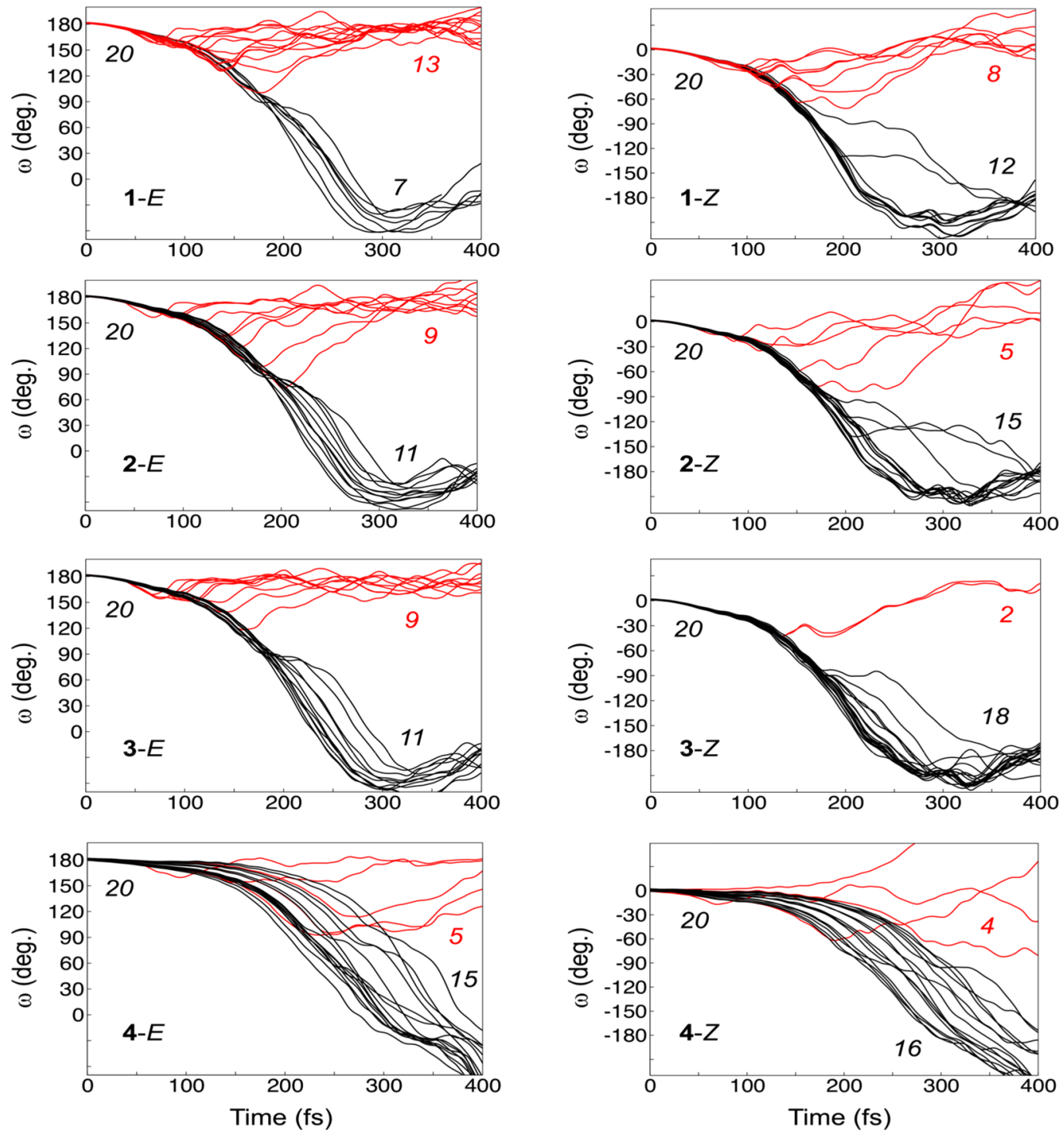

Figure 3. Photoinduced torsional motion around the central olefinic bond during the first $400 \mathrm{fs}$ of all $20 \mathrm{NAMD}$ trajectories run for each of the $E$ and $Z$ isomers of 1-4. In each panel, the two values to the right indicate the number of successful (black font) and unsuccessful (red font) trajectories that undergo and fail to undergo a complete photoisomerization, respectively, within 800 fs. Notice that the successful trajectories of 1-3 are faster than those of 4 (see also Table S5), but have the same overall shape.

that were calculated for the cyclopentadiene motif of the $E$ and $Z$ isomers of 1-3 along the MEPs. These results, which are discussed in the Supporting Information (in connection to Figure S2), clearly show that this motif becomes aromatic as the $E / Z$ photoisomerizations proceed in the $S_{2}$ state, as a consequence of electron donation from the $N$-methylpyrrolidine moiety and heterolytic $\pi$-bond cleavage (see Figure 1a).

Although the MEP calculations reveal a propensity for 1-3 to undergo $E / Z$ photoisomerizations, the static nature of these calculations and their reliance on the adiabatic BO approximation preclude an assessment of what role the isotopic chirality of $\mathbf{2}$ and 3 might play for the reactions. In particular, the MEP calculations do not provide any information about whether the isotopically chiral $\mathbf{2}$ and $\mathbf{3}$ are better able than the achiral $\mathbf{1}$ to achieve the asymmetry that would allow consecutive $E \rightarrow Z$ and $Z \rightarrow E$ photoisomerizations to produce a full, unidirectional $360^{\circ}$ rotation around the central olefinic bond. Importantly, it is this very characteristic that is the hallmark of synthetic rotary molecular motors. ${ }^{27}$
To address this issue, NAMD simulations were carried out by using Tully's fewest switches algorithm ${ }^{28}$ with the CASSCF method and the 6-31G(d) basis set, as further detailed in the Supporting Information. Simulations of this type, which have been reported for a number of different molecular motors, $7,20 a, 29$ describe the simultaneous dynamics of electronic (treated quantum mechanically) and nuclear (treated classically) degrees of freedom during photochemical reactions. Accordingly, they are sensitive to nuclear masses and can probe isotope effects on such reactions (see, for example, studies of isotope effects on excited-state proton transfer reactions by Thiel and coworkers $^{30}$ ). The simulations were started in the $S_{2}$ state of the $E$ and $Z$ isomers of $\mathbf{1 - 3}$ and were run for maximally $800 \mathrm{fs}$ with 20 different initial nuclear configurations and velocities for each species. For reference purposes, simulations were also performed for the $E$ and $Z$ isomers of the chemically chiral motor design 4 . The results of all 160 simulations are presented in Figure 3. 
Defining a successful NAMD trajectory as one that reaches the $S_{0}$ state and completes a photoisomerization by undergoing a full $180^{\circ}$ rotation around the central olefinic bond within $800 \mathrm{fs}$, one can first see that the ratio of successful trajectories shown by 4 is high, amounting to 31 of 40 . Furthermore, all of these 31 trajectories are perfectly unidirectional, with the associated rotations exclusively occurring toward decreasing $\omega$ values. Thus, 4 has the same ability as many different synthetic molecular motors ${ }^{1-4}$ to produce a full $360^{\circ}$ rotation from two consecutive $E \rightarrow Z$ and $Z \rightarrow E$ photoisomerizations. As for the nine unsuccessful trajectories, these include situations in which the photoexcited system remains in the $S_{2}$ state without isomerizing, or in which decay to the $S_{0}$ state is followed by re-formation of the starting isomer.

Turning to the results for 1 , the $19 / 40$ ratio of successful trajectories for this achiral species is markedly lower than that attained by $4(31 / 40)$. This illustrates how the chemical chirality of 4 introduces the asymmetry needed for the overall excitedstate dynamics of its $E$ and $Z$ isomers to better sustain unidirectional rotary motion. However, continuing with the results for $\mathbf{2}$ and 3, it is remarkable that the isotopic chirality of these species realizes the very same objective to an almost comparable degree: for $2\left({ }^{2} \mathrm{H} /{ }^{1} \mathrm{H}\right.$ chiral $)$, the ratio is $26 / 40$, and for $3\left({ }^{3} \mathrm{H} /{ }^{1} \mathrm{H}\right.$ chiral $)$, it is $29 / 40$. Moreover, 2 and 3 photoisomerize faster than 4 (see also Table S5), which is likely due to the smaller moments of inertia with respect to the rotation axis along the central olefinic bond that come from replacing the $\mathrm{C} 5$ methyl group of 4 with ${ }^{2} \mathrm{H}$ and ${ }^{3} \mathrm{H}$ in 2 and 3 , respectively. Taken together, the results in Figure 3 identify a previously unknown but potential key role for isotopic chirality in the future development of light-driven rotary molecular motors. Furthermore, and more generally, the findings show that the control of asymmetric autocatalysis ${ }^{14 c, 15-18}$ and deracemization processes ${ }^{31}$ that isotopic chirality has proven able to afford, also extends to an isomerization reaction ubiquitous in organic chemistry.

\section{ASSOCIATED CONTENT}

\section{Supporting Information}

The Supporting Information is available free of charge at https://pubs.acs.org/doi/10.1021/acs.orglett.0c02436.

Computational details, complementary results (molecular orbitals in Figure S1, aromaticity indices in Figure S2, excitation energies in Table S1, orbital occupation numbers in Table S2, geometric parameters in Tables S3 and S4, and average photoisomerization times in Table S5), and Cartesian coordinates and energies of different geometries of $\mathbf{1 - 4}$ (PDF)

Animation of two representative trajectories from the NAMD simulations of 3 (one for the $E$ isomer and one for the $Z$ isomer) merged together to illustrate a full $360^{\circ} \mathrm{E} \rightarrow$ $Z \rightarrow E$ rotation around the central olefinic bond (MP4)

\section{AUTHOR INFORMATION}

\section{Corresponding Authors}

Jun Wang - Division of Theoretical Chemistry, IFM, Linköping University, SE-58183 Linköping, Sweden; Institut de Quimica Computacional i Catàlisi, Facultat de Ciencies, Universitat de Girona, ES-17003 Girona, Spain; 이이이.org/0000-00030222-6380; Email: jun.wang@udg.edu
Bo Durbeej - Division of Theoretical Chemistry, IFM, Linköping

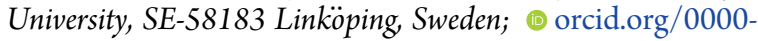
0001-5847-1196; Email: bodur@ifm.liu.se

Author

Baswanth Oruganti - Department of Chemistry and Biomedical Sciences, Faculty of Health and Life Sciences, Linnaeus University, SE-45041 Kalmar, Sweden; 이이.org/0000-0002-41992750

Complete contact information is available at: https://pubs.acs.org/10.1021/acs.orglett.0c02436

\section{Notes}

The authors declare no competing financial interest.

\section{ACKNOWLEDGMENTS}

B.D. acknowledges financial support from the Olle Engkvist Foundation (Grants 184-568 and 204-0183) and the Swedish Research Council (Grant 2019-03664), and grants of computing time at the National Supercomputer Centre (NSC) in Linköping.

\section{REFERENCES}

(1) (a) Koumura, N.; Zijlstra, R. W. J.; van Delden, R. A.; Harada, N.; Feringa, B. L. Light-Driven Monodirectional Molecular Rotor. Nature 1999, 401, 152-155. (b) Koumura, N.; Geertsema, E. M.; van Gelder, M. B.; Meetsma, A.; Feringa, B. L. Second Generation Light-Driven Molecular Motors. Unidirectional Rotation Controlled by a Single Stereogenic Center with Near-Perfect Photoequilibria and Acceleration of the Speed of Rotation by Structural Modification. J. Am. Chem. Soc. 2002, 124, 5037-5051.

(2) (a) Guentner, M.; Schildhauer, M.; Thumser, S.; Mayer, P.; Stephenson, D.; Mayer, P. J.; Dube, H. Sunlight-Powered kHz Rotation of a Hemithioindigo-Based Molecular Motor. Nat. Commun. 2015, 6, 8406. (b) Gerwien, A.; Mayer, P.; Dube, H. Green Light Powered Molecular State Motor Enabling Eight-Shaped Unidirectional Rotation. Nat. Commun. 2019, 10, 4449.

(3) Everhart, S. C.; Jayasundara, U. K.; Kim, H.; Procúpez-Schtirbu, R.; Stanbery, W. A.; Mishler, C. H.; Frost, B. J.; Cline, J. I.; Bell, T. W. Synthesis and Photoisomerization of Substituted Dibenzofulvene Molecular Rotors. Chem. - Eur. J. 2016, 22, 11291-11302.

(4) (a) Greb, L.; Lehn, J.-M. Light-Driven Molecular Motors: Imines as Four-Step or Two-Step Unidirectional Rotors. J. Am. Chem. Soc. 2014, 136, 13114-13117. (b) Greb, L.; Eichhöfer, A.; Lehn, J.-M. Synthetic Molecular Motors: Thermal N Inversion and Directional Photoinduced $\mathrm{C}=\mathrm{N}$ Bond Rotation of Camphorquinone Imines. Angew. Chem., Int. Ed. 2015, 54, 14345-14348.

(5) Kistemaker, J. C. M.; Stacko, P.; Visser, J.; Feringa, B. L. Unidirectional Rotary Motion in Achiral Molecular Motors. Nat. Chem. 2015, 7, 890-896.

(6) (a) Richter, M.; Marquetand, P.; González-Vázquez, J.; Sola, I.; González, L. SHARC: ab Initio Molecular Dynamics with Surface Hopping in the Adiabatic Representation Including Arbitrary Couplings. J. Chem. Theory Comput. 2011, 7, 1253-1258. (b) Barbatti, M. Nonadiabatic Dynamics with Trajectory Surface Hopping Method. WIREs Comput. Mol. Sci. 2011, 1, 620-633.

(7) Wang, J.; Oruganti, B.; Durbeej, B. Light-Driven Rotary Molecular Motors without Point Chirality: A Minimal Design. Phys. Chem. Chem. Phys. 2017, 19, 6952-6956.

(8) (a) Pérez-Hernández, G.; Pelzer, A.; González, L.; Seideman, T. Biologically Inspired Molecular Machines Driven by Light. Optimal Control of a Unidirectional Rotor. New J. Phys. 2010, 12, 075007. (b) Zhang, J.; Sergeeva, A. P.; Sparta, M.; Alexandrova, A. N. $\mathrm{B}_{13}{ }^{+}$: A Photodriven Molecular Wankel Engine. Angew. Chem., Int. Ed. 2012, $51,8512-8515$ 
(9) (a) Arigoni, D.; Eliel, E. L. Chirality Due to the Presence of Hydrogen Isotopes at Noncyclic Positions. In Topics in Stereochemistry; Allinger, N. L., Eliel, E. L., Eds.; John Wiley \& Sons, Inc.: New York, 1969; Vol. 4, pp 127-243. (b) Berger, R.; Laubender, G.; Quack, M.; Sieben, A.; Stohner, J.; Willeke, M. Isotopic Chirality and Molecular Parity Violation. Angew. Chem., Int. Ed. 2005, 44, 3623-3626. (c) Barabás, B.; Caglioti, L.; Micskei, K.; Zucchi, C.; Pályi, G. Isotope Chirality and Asymmetric Autocatalysis: A Possible Entry to Biological Chirality. Origins Life Evol. Biospheres 2008, 38, 317-327.

(10) Eliel, E. L. The Reduction of Optically Active Phenylmethylcarbinyl Chloride with Lithium Aluminum Deuteride. J. Am. Chem. Soc. 1949, 71, 3970-3972.

(11) Barth, G.; Djerassi, C. Circular Dichroism of Molecules with Isotopically Engendered Chirality. Tetrahedron 1981, 37, 4123-4142.

(12) Dierksen, M.; Grimme, S. A Theoretical Study of the Chiroptical Properties of Molecules with Isotopically Engendered Chirality. J. Chem. Phys. 2006, 124, 174301.

(13) (a) Cantekin, S.; Balkenende, D. W. R.; Smulders, M. M. J.; Palmans, A. R. A.; Meijer, E. W. The Effect of Isotopic Substitution on the Chirality of a Self-Assembled Helix. Nat. Chem. 2011, 3, 42-46. (b) Nakano, Y.; Markvoort, A. J.; Cantekin, S.; Filot, I. A. W.; ten Eikelder, H. M. M.; Meijer, E. W.; Palmans, A. R. A. Conformational Analysis of Chiral Supramolecular Aggregates: Modeling the Subtle Difference between Hydrogen and Deuterium. J. Am. Chem. Soc. 2013, 135, 16497-16506.

(14) (a) Soai, K.; Shibata, T.; Morioka, H.; Choji, K. Asymmetric Autocatalysis and Amplification of Enantiomeric Excess of a Chiral Molecule. Nature 1995, 378, 767-768. (b) Blackmond, D. G. Asymmetric Autocatalysis and Its Implications for the Origin of Homochirality. Proc. Natl. Acad. Sci. U. S. A. 2004, 101, 5732-5736.

(c) Hawbaker, N. A.; Blackmond, D. G. Rationalization of Asymmetric Amplification via Autocatalysis Triggered by Isotopically Chiral Molecules. ACS Cent. Sci. 2018, 4, 776-780. (d) Soai, K. Asymmetric Autocatalysis. Chiral Symmetry Breaking and the Origins of Homochirality of Organic Molecules. Proc. Jpn. Acad., Ser. B 2019, 95, 89-110.

(15) Kawasaki, T.; Shimizu, M.; Nishiyama, D.; Ito, M.; Ozawa, H.; Soai, K. Asymmetric Autocatalysis Induced by Meteoritic Amino Acids with Hydrogen Isotope Chirality. Chem. Commun. 2009, 4396-4398.

(16) Kawasaki, T.; Matsumura, Y.; Tsutsumi, T.; Suzuki, K.; Ito, M.; Soai, K. Asymmetric Autocatalysis Triggered by Carbon Isotope $\left({ }^{13} \mathrm{C} /{ }^{12} \mathrm{C}\right)$ Chirality. Science 2009, 324, 492-495.

(17) Kawasaki, T.; Okano, Y.; Suzuki, E.; Takano, S.; Oji, S.; Soai, K. Asymmetric Autocatalysis: Triggered by Chiral Isotopomer Arising from Oxygen Isotope Substitution. Angew. Chem., Int. Ed. 2011, 50, $8131-8133$.

(18) Matsumoto, A.; Ozaki, H.; Harada, S.; Tada, K.; Ayugase, T.; Ozawa, H.; Kawasaki, T.; Soai, K. Asymmetric Induction by a Nitrogen ${ }^{14} \mathrm{~N} /{ }^{15} \mathrm{~N}$ Isotopomer in Conjunction with Asymmetric Autocatalysis. Angew. Chem., Int. Ed. 2016, 55, 15246-15249.

(19) Miura, T.; Nakamuro, T.; Nagata, Y.; Moriyama, D.; Stewart, S. G.; Murakami, M. Asymmetric Synthesis and Stereochemical Assignment of ${ }^{12} \mathrm{C} /{ }^{13} \mathrm{C}$ Isotopomers. J. Am. Chem. Soc. 2019, 141, 1334113345.

(20) (a) Oruganti, B.; Wang, J.; Durbeej, B. Excited-State Aromaticity Improves Molecular Motors: A Computational Analysis. Org. Lett. 2017, 19, 4818-4821. (b) Durbeej, B.; Wang, J.; Oruganti, B. Molecular Photoswitching Aided by Excited-State Aromaticity. ChemPlusChem 2018, 83, 958-967.

(21) (a) Conyard, J.; Addison, K.; Heisler, I. A.; Cnossen, A.; Browne, W. R.; Feringa, B. L.; Meech, S. R. Ultrafast Dynamics in the Power Stroke of a Molecular Rotary Motor. Nat. Chem. 2012, 4, 547-551. (b) Conyard, J.; Cnossen, A.; Browne, W. R.; Feringa, B. L.; Meech, S. R. Chemically Optimizing Operational Efficiency of Molecular Rotary Motors. J. Am. Chem. Soc. 2014, 136, 9692-9700.

(22) Andersson, K.; Malmqvist, P.-Å.; Roos, B. O. Second-Order Perturbation Theory with a Complete Active Space Self-Consistent Field Reference Function. J. Chem. Phys. 1992, 96, 1218-1226.
(23) Christiansen, O.; Koch, H.; Jørgensen, P. The Second-Order Approximate Coupled Cluster Singles and Doubles Model CC2. Chem. Phys. Lett. 1995, 243, 409-418.

(24) Roos, B. O.; Taylor, P. R.; Sigbahn, P. E. M. A Complete Active Space SCF Method (CASSCF) Using a Density Matrix Formulated Super-CI Approach. Chem. Phys. 1980, 48, 157-173.

(25) Krygowski, T. M. Crystallographic Studies of Inter- and Intramolecular Interactions Reflected in Aromatic Character of $\pi$ Electron Systems. J. Chem. Inf. Model. 1993, 33, 70-78.

(26) Noorizadeh, S.; Shakerzadeh, E. Shannon Entropy as a New Measure of Aromaticity, Shannon Aromaticity. Phys. Chem. Chem. Phys. 2010, 12, 4742-4749.

(27) (a) Kay, E. R.; Leigh, D. A.; Zerbetto, F. Synthetic Molecular Motors and Mechanical Machines. Angew. Chem., Int. Ed. 2007, 46, 72191. (b) Feringa, B. L. The Art of Building Small: From Molecular Switches to Motors (Nobel Lecture). Angew. Chem., Int. Ed. 2017, 56, 11060-11078.

(28) Tully, J. C. Molecular Dynamics with Electronic Transitions. J. Chem. Phys. 1990, 93, 1061-1071.

(29) (a) Marchand, G.; Eng, J.; Schapiro, I.; Valentini, A.; Frutos, L. M.; Pieri, E.; Olivucci, M.; Léonard, J.; Gindensperger, E. Directionality of Double-Bond Photoisomerization Dynamics Induced by a Single Stereogenic Center. J. Phys. Chem. Lett. 2015, 6, 599-604. (b) Filatov, M.; Paolino, M.; Min, S. K.; Kim, K. S. Fulgides as Light-Driven Molecular Rotary Motors: Computational Design of a Prototype Compound. J. Phys. Chem. Lett. 2018, 9, 4995-5001. (c) Wang, J.; Durbeej, B. Toward Fast and Efficient Visible-Light-Driven Molecular Motors: A Minimal Design. ChemistryOpen 2018, 7, 583-589. (d) Wang, J.; Oruganti, B.; Durbeej, B. A Straightforward Route to Aromatic Excited States in Molecular Motors that Improves Photochemical Efficiency. ChemPhotoChem. 2019, 3, 450-460.

(30) Spörkel, L.; Cui, G.; Koslowski, A.; Thiel, W. Nonequilibrium H/ D Isotope Effects from Trajectory-Based Nonadiabatic Dynamics. J. Phys. Chem. A 2014, 118, 152-157.

(31) Murray, J. I.; Sanders, J. N.; Richardson, P. F.; Houk, K. N.; Blackmond, D. G. Isotopically Directed Symmetry Breaking and Enantioenrichment in Attrition-Enhanced Deracemization. J. Am. Chem. Soc. 2020, 142, 3873-3879. 